\title{
Light Sheet Fluorescence Microscopy (LSFM)
}

Michael W. Adams, Andrew F. Loftus, Sarah E. Dunn, Matthew S. Joens, and James A.J. Fitzpatrick*

Waitt Advanced Biophotonics Center, Salk Institute for Biological Studies, 10010 North Torrey

Pines Road, La Jolla, CA 92037

\section{Abstract}

The development of confocal microscopy techniques introduced the ability to optically section fluorescent samples in the axial dimension, perpendicular to the image plane. These approaches, via the placement of a pinhole in the conjugate image plane, provided superior resolution in the axial (z) dimension resulting in nearly isotropic optical sections. However, increased axial resolution, via pinhole optics, comes at the cost of both speed and excitation efficiency. Light Sheet Fluorescent Microscopy (LSFM), a century old idea (Siedentopf and Zsigmondy, 1902) made possible with modern developments in both excitation and detection optics, provides subcellular resolution and optical sectioning capabilities without compromising speed or excitation efficiency. Over the past decade, several variations of LSFM have been implemented each with its own benefits and deficiencies. Here we discuss LSFM fundamentals and outline the basic principles of several major light sheet based imaging modalities (SPIM, inverted SPIM, multiview SPIM, Bessel beam SPIM, and stimulated emission depletion SPIM while considering their biological relevance in terms of intrusiveness, temporal resolution, and sample requirements.

\section{Keywords}

Light Sheet Fluorescence Microscopy (LSFM); Selective Plane Illumination Microscopy (SPIM); Inverted Selective Plane Illumination Microscopy (iSPIM); Multi-view Selective Plane Illumination Microscopy (mSPIM); Bessel Beam Super-Resolution Structured Illumination Microscopy (BB-SR-SIM); Stimulated Emission Depletion Selective Plane Illumination Microscopy (STED-SPIM); 3D imaging; 4D imaging; developmental imaging; embryogenesis

\section{Introduction}

Optical sectioning is the primary purpose of confocal microscopy techniques. Traditional galvanometric-based point scanning confocal microscopes (the most widely used confocal modality), are typically limited in live cell applications due to their slow acquisition speeds and repeated exposure of the sample to the excitation light intensity. Confocal imaging speeds have subsequently improved to near video rate acquisition with the introduction of the Nipkow spinning disk (Nakano, 2002) which makes use of two co-rotating disks, one of microlenses and one of pinholes spinning at high revolutions to acquire an array of pixels simultaneously. Imaging times have been reduced even further with the advent of more

\footnotetext{
`Correspondence should be addressed to: fitzp@salk.edu.
} 
sensitive detectors such as electron multiplied CCD (EM-CCD) and Scientific

Complementary Metal-Oxide Semiconductor (sCMOS) cameras allowing shorter acquisition times on the millisecond regime. More recent advances in point scanning microscopes have given rise to both resonant (Hendriks et al., 2011) and programmable array scanning (PAM) (Hanley et al., 1999) capabilities which, when combined with recent advances in detectors such as Hybrid (Michalet et al., 2008) or GaAsP (Becker et al., 2011) photomultipliers (PMTs), lead to acquisition speeds that are nearly comparable to spinning disk systems. However, since all of these confocal modalities optically section via pinhole optics in the emission light path, they do nothing to reduce the sample's exposure to illumination intensity as they must repeatedly illuminate large out of focus regions within the sample (see Figure 1A-C). They are thus the least efficient means of excitation for obtaining optically sectioned $2 \mathrm{D}, 3 \mathrm{D}$, or 4D image datasets.

In contrast, Light Sheet Fluorescence Microscopy (LSFM) synonymous with Selective Plane Illumination Microscopy (SPIM), optically sections a sample via the excitation light path (see Figure 1D). Utilizing mutually orthogonal planes for excitation and emission, LSFM offers high speed acquisition, efficient excitation, and an improved depth of field while retaining subcellular resolution and an ability to optically section in the axial dimension. LSFM improves excitation efficiency, with a reduction of up to three orders of magnitude (Keller and Stelzer, 2008), by only illuminating the focal plane of the imaging objective during each acquisition. This facilitates imaging of living samples by reducing photodamage and thereby providing a less invasive modality. Furthermore, by illuminating the entire focal plane simultaneously, LSFM is able to take advantage of modern sCMOS cameras to acquire the entire field of view of the imaging objective, at high resolution, and with a short exposure time. This reduces not only the cumulative dose of light to the sample, but also the fractioned dose (acute exposure); thereby allowing living samples to recover thus improving sample viability. Additionally, LSFM systems that employ identical objectives for both sheet illumination and imaging have the additional benefit of acquiring 3D volumes from more than one angle allowing tomographic-like reconstruction of the volume yielding higher resolution and isotropic data sets (Buytaert et al., 2012; Huisken and Stainier, 2007).

Since the first modern LSFM system was described by Voie et al. there have been several variations described in the literature (Voie et al., 1993). In a broad sense, these variations center on how the light sheet is created and how the three-dimensional volume is acquired and reconstructed. One advance has greatly broadened the potential breadth of LSFM samples by creating an inverted system allowing more traditional sample mounting such as adherent cell cultures. More recently, light sheet based imaging approaches have successfully been coupled with super-resolution imaging paradigms which allow imaging beyond the diffraction limit across the entire illumination plane. Ultimately, how the sheet is engineered has the greatest effect on the technical limitations and benefits of each variation of the LSFM modality. 


\section{Light Sheet Fluorescence Microscopy (LSFM)}

\section{Basic principles of design}

Nearly a century after the first published account of orthogonal light sheet microscopy by Siedentopf and Zsigmondy (Siedentopf and Zsigmondy, 1902), Voie et al. reported the first use of light sheet microscopy for fluorescent imaging. Light sheet excitation was employed to image the internal architecture of the gineau pig cochlea using, what they termed, Orthogonal Plane Fluorescence Optical Sectioning (OPFOS) (Voie et al., 1993). More recently, Fuchs et al. reported a Thin Light Sheet Microscope (TLSM) for imaging bacterial content in seawater (Fuchs et al., 2002) and Huiskens et al. reported imaging Drosophila embryogenesis using a technique called Selective Plane Illumination Microscopy (SPIM) (Huisken et al., 2004). What all of these basic modern LSFM systems have in common is the employment of an imaging objective and an orthogonal cylindrical lens or excitation objective that excites the fluorescent sample with a thin sheet of light. The emission is collected with a second objective and recorded by either an EM-CCD or SCMOS camera. Since optical sectioning is provided by the excitation light sheet, pinhole optics are unnecessary thereby allowing LSFM to image the entire focal plane simultaneously. With orthogonal excitation and imaging planes, LSFM drastically improves imaging speeds while minimizing photodamage to living samples (Huisken et al., 2004). Compared to point scanning confocal microscopes, using the same imaging objective magnification, orthogonal illumination gives greater depth of field for samples that are symmetrical about two dimensions. The LSFM modality can be thought of as three distinct components working in unison; (i) excitation light sheet, (ii) emission detection and (iii) sample orientation.

\section{Excitation Light Sheet}

The basic LSFM or SPIM modality differs from that of traditional epifluorescence widefield microscopy almost entirely by means of sample excitation. Rather than illuminating the entirety of the sample, LSFM utilizes an orthogonal light sheet to excite only a sub region of the sample within the focal plane of the imaging objective. In the simplest case of orthogonal LSFM, the excitation light sheet can be generated via two methods (i) a cylindrical lens is placed after a square aperture to generate a sheet of light from an excitation source that is orthogonal to the image plane or (ii) a galvanometric mirror is used to generate a line scanned sheet in the orthogonal plane through an excitation objective (see Figure 2A). Since the divergence of the light sheet from the focal point is largely dependent on the numerical aperture (NA) of the excitation objective or cylindrical lens, it can be beneficial to use lower NA optics to improve the homogeneity of the sheet across the illumination plane. Cylindrical lenses offer a lower NA compared to a traditional microscope objective, providing a greater homogeneity of excitation over a larger field of view. The maximum desired field of view can be approximated by the confocal parameter $C_{p}$ as shown in Equation 1:

$$
C_{P}=2 Z_{r}=\frac{2 \pi \omega_{o}^{2}}{\lambda} \cong \frac{2 \omega_{o}}{N A} \quad \text { Equation } 1
$$


where $Z_{r}$ is the Rayleigh length, $\lambda$ is the excitation wavelength, $\omega_{0}$ is the beam waist at the thinnest point, and NA is the beam waist at the is the numerical aperture of the excitation lens. The confocal parameter, also called the depth of focus, is a theoretical measure of the distance between the two points about the beam waist $\left(\omega_{0}\right)$, in which the depth of the light sheet increases to $\sqrt{2} \omega_{o}$ (see Figure 2B). One caveat of using a lower NA optic, such as a cylindrical lens, is that the minimum beam waist of the excitation sheet is generally larger since it, too, is inversely proportional to the NA of the excitation lens (Santi et al., 2009) and results in a lower axial (z) resolution as shown in Equation 2:

$$
\omega_{o} \cong \frac{\lambda}{\pi N A} \quad \text { Equation } 2
$$

An additional caveat to using a solid light sheet across a thick sample is that unwanted striping artifacts occur. In contrast, the second (galvo-based line scanning) method can be used to minimize the excitation volume and thus improve the axial (z) resolution via the use of higher NA objectives. Furthermore, the striping artifact, typical of cylindrical lens based LSFM, is reduced via line scanning and intentional addition of yaw to the incident beam. This method, however does suffer from a non-homogeneous excitation sheet over a large field of view, but these effects can be minimized by reducing the field of view of the imaging objective. This can be achieved by using a modest NA (eg. 10X 0.3NA) excitation objective and a higher NA (eg. 40x 1.2NA) imaging objective.

\section{Emission Detection and Image Collection}

For basic LSFM, the imaging/emission light path is nearly identical to that of the epifluorescence widefield imaging modality. Although the light paths are nearly identical, LSFM has more restrictions on the choice of imaging objective. Choosing an appropriate objective is a matter of matching the working distance to the sample holder size and the field of view to the confocal parameter defined by the light sheet characteristics shown in Equation 1 above.

Once an appropriate imaging objective is chosen, sample fluorescence is detected using an emission filter, tube lens and camera (see Figure 2A). Much like a widefield microscope, the final resolution of the image is typically limited by the resolution of the camera itself. For this reason, most modern light sheet fluorescent microscopes use sCMOS cameras for their high spatial (small pixel sixe) and temporal resolution (high frame rate). Although EMCCD cameras provide a better level of sensitivity, and therefore faster acquisition times, they are typically limited in spatial resolution to $512 \times 512$ pixels for video rate acquisition. In contrast, sCMOS offers approximately $2560 \times 2160$ pixel resolution while retaining sufficient sensitivity for acquisition times of 100 frames per second.

\section{Sample Orientation and Preparation}

The basic LSFM design has both benefits and limitations on sample preparation due to the geometry of the orthogonal imaging and excitation planes. The benefit, of course, is the ability to image much larger samples in 3D/4D than other more traditional fluorescence microscopy modalities. Sample preparation techniques for traditional fluorescent 
microscopy modalities have only one spatial requirement i.e. that the sample be within the working distance of the imaging objective (axial distance). LSFM shares this requirement along the axial dimension, but has the additional constraint that the sample also be within the working distance of the excitation objective. This limitation requires samples to be mounted/suspended in a manner which minimizes sample distance in not just one, but two orthogonal dimensions while maintaining light sheet integrity. Therefore, LSFM samples are typically suspended in a small water, media, or agarose-filled cylindrical chamber that provides equal sample-chamber distance in two of the three spatial dimensions (see Figure 2C-D). In addition, the cylindrical sample holder provides an ideal geometry for sample rotation, allowing tomographic imaging and reconstruction. This is largely beneficial for much thicker samples that would suffer from uneven illumination were the sample to be illuminated from only one view.

Unfortunately, this cuvette style sample holder restricts basic LSFM samples to those which have at least two symmetric spatial dimensions and do not require a flat membrane or surface for attachment. Furthermore, since the optical sectioning of the sample is typically accomplished by moving the sample rather than the objectives, the sample must also be mounted in such a way as to prevent sample movement during translation and/or rotation. Developing embryos typically fit within these constraints easily, and therefore have been the primary subject of study for the basic LSFM modality to date. For example, LSFM has been successfully employed to map cell dynamics during early embryonic development in the zebrafish (Keller et al., 2008) and tracing cell lineage (Swoger et al., 2011). Sample mounting is a critical challenge for long-term developmental studies. Hence, multi-layer mounting approaches have been developed to ensure stable conditions, such as multi-layer approach applied by (Kaufmann et al., 2012) for imaging developing zebrafish embryos. LSFM has also been used to image the development of $C$. elegans embryos (Chardes et al., 2014), amoeboid movements (Takao et al., 2012), bacterial symbioses in developing zebrafish larvae (Taormina et al., 2012) and development of Tribolium castaneum embryos (Strobl and Stelzer, 2014). Despite the overwhelming focus on embryonic development, LSFM has also been used to image large cellular specimens such as three-dimensional

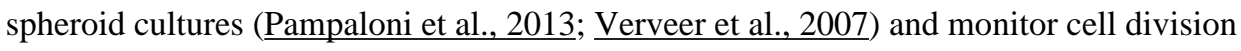
dynamics within large spheroid tumor models (Lorenzo et al., 2011).

In contrast, any living sample that requires a surface on which to adhere or grow does not benefit from this approach since the center, and region of highest imaging quality would be wasted. Since typical micro-fluidic devices require photolithography for precise engineering, they too are eliminated from the basic LSFM modality as they require a flat surface for the photomask. Maskless lithography, for example electron beam lithography, or 3D printing technologies could be used to work around this limitation. Fortunately, the inverted Selective Plane Illumination Microscopy (iSPIM) approach, is a natural extension of the basic LSFM modality that allows for more traditional (single view) sample preparation techniques such as adherent cell monolayers and organisms housed in microfluidic devices. 


\section{Inverted Selective Plane Illumination Microscopy (iSPIM)}

Given the sample limitations and custom, often sample specific, sample holder arrangements of the basic LSFM approach, a new technique was necessary to expand the LSFM imaging modality for more flexible sample mounting. Inverted Selective Plane Illumination Microscopy (iSPIM), introduced in 2011 (Wu et al., 2011) offers more traditional sample mounting, along with a potential reduction in cost, by making use of a regular inverted microscope platform. The inverted design provides live imaging capabilities of the LSFM modality while retaining the flexibility of inverted imaging systems. iSPIM replaces the transmitted light pillar (above the stage) with a motorized housing containing excitation and imaging objectives in an orthogonal orientation for light sheet excitation and fluorescent imaging. In order to image without moving the sample, iSPIM employs the second method of light sheet engineering (line scanning) discussed above. Additionally, the inverted configuration allows for a third (inverted) objective placed below the sample for quick focus finding or as a condenser for transmitted light imaging if desired (see Figure 3).

In order to maintain the necessary $90^{\circ}$ (orthogonal) orientation of the excitation and emission objectives, the iSPIM setup employs non-traditional objective-sample orientation angles of $45^{\circ}$. While this objective-sample geometry could be successfully incorporated into the basic LSFM design, iSPIM offers several unique advantages: (i) aided by gravity, upright imaging is ideal for using immersion media; (ii) the traditional stage top sample holder is far more versatile, hence allowing a broader range of mounting techniques and (iii) sample settling does not occur since the sample is already in a resting state rather than suspended in a mounting medium such as agarose. While inverting the LSFM modality is relatively straightforward, it has greatly amended the breadth, ease of use, and reproducibility of the LSFM approach.

As with any other imaging modality, iSPIM naturally trades improvements with some diminished capabilities. The most profound of which, is the loss of working distance due to the objective-sample orientation. The loss of working distance varies depending on the objective used (size of front lens and housing), as the objective housing meets the sample prior to the optical surface of the lens. Wu et al. chose two 40x (0.8NA) water immersion objectives as they provided maximal resolution and minimized refractive index mismatch while staying within the physical limitations of the desired translation steps required for $C$. elegans neurodevelopmental imaging (Wu et al., 2011). The choice of objectives is further complicated since they must not interfere with the sample or with one another. While this is also true for orthogonal LSFM techniques, this problem is exasperated by the $45^{\circ}$ working geometry at the objective-sample interface. While coverslip mounted samples are easier to image with iSPIM as compared to basic LSFM, the light sheet and focal planes are not normal to the coverslip interface. This introduces unwanted optical aberrations that are not easy to correct. Moreover, this will result in a more complex Gaussian beam broadening than what is described by the equations above, therefore leaving a smaller effective field of view.

iSPIM is ideally suited for neurodevelopmental applications because of the upright imaging configuration and minimized sample intrusiveness. Although published applications are 
limited to those of developing nematodes and living cells (Wu et al., 2011; Wu et al., 2013), the inverted objective-sample arrangement could be successfully extended to in vivo studies of larger organisms. No longer requiring a coverslip, whole brain/organ in vivo imaging would reduce the aberrations caused by non-normal incident light through the coverslip. In addition, no coverslip requirement, increases the potential working depth of the system by allowing the objectives to be placed closer to the sample. Sample opacity would, of course, play a major role in the final effective working distance of the system. The inverted arrangement is also ideal for experiments requiring microfluidic devices for high throughput neurological studies of nematodes. As noted by Wu et al. (Wu et al., 2011), iSPIM extends naturally to dual view (dvSPIM) imaging arrangements that yield higher-resolution isotropic volumes.

\section{Multi-view Selective Plane Illumination Microscopy (mSPIM)}

Multi-view Selective Plane Illumination Microscopy (mSPIM) utilizes multiple views of the sample in order to reconstruct a three dimensional volume. mSPIM techniques can be segregated into three methodologies: (i) dual-excitation (deSPIM) (Ahrens et al., 2013; Huisken and Stainier, 2007); (ii) rotation SPIM (rmSPIM) (Huisken et al., 2004); and (iii) dual-view SPIM (dvSPIM) (Wu et al., 2013). These three methods differ primarily by temporal and spatial resolution, each trading one for another. They all share several significant improvements, unique to the multi-view modality, including: improved illumination homogeneity, larger sample sizes, increased axial resolution, and enhanced signal to noise response.

Imaging large or opaque samples, with LSFM, often results in unwanted attenuation of the excitation sheet that escalates with depth of penetration. This results in, often visible, striping artifacts caused by shadows from punctate high attenuation regions. These artifacts lead to loss of information, subsequently reducing the effective resolution of the system. These artifacts can be reduced by the dual excitation (deSPIM) method, by illuminating the sample with not one, but two excitation sheets $\left(180^{\circ}\right.$ separated) from opposing views, but with a single imaging objective (see Figure 4A). Overall sample attenuation, whether it is due to depth or opacity of the sample, is effectively halved resulting in the ability to image samples twice as large as previously discussed. By appropriately aligning the focal points of the two separate excitation objectives, dual excitation gives the option of a larger field of view, or improved axial resolution via thinner optical sectioning. To achieve a larger field of view, offset the focal points of the excitation objectives by a distance equal to the confocal parameter and excite sequentially. This technique will double the effective field of view size (see Equation 1). To improve axial resolution, co-align the excitation objectives and keep the field of view of the emission collection equal to one confocal parameter length and excite sequentially. This setup allows for thinner sheet illumination by a factor of $\sqrt{2}$ (see Equation 2). Sequential imaging requires that the images be registered during a postprocessing step. There are several correlative approaches for automatic alignment, but Huisken and Swoger address it in their studies (Swoger et al., 2003; Swoger et al., 2007). Additionally, the dual light sheet geometry reduces the effects of scattering, hence increasing signal to noise response at depth, since each light sheet need only penetrate half of the sample. For example Ahrens et al. used the dePSIM approach to image neural activity 
using the GCaMP5G calcium sensor in the developing brain of an intact larval zebrafish at $0.8 \mathrm{~Hz}$ (Ahrens et al., 2013). In the line scanning case, along with opposing excitation objectives, Huisken et al. incorporated a small $10^{\circ}$ line rotation with a $1 \mathrm{kHz}$ resonant mirror. This fast sheet rotation (yaw) greatly reduced striping artifacts (Huisken and Stainier, 2007).

Sample rotation (rmSPIM), the second multi-view technique, offers many of the same benefits of dual light sheet excitation while adding additional imaging perspectives, allowing for more accurate reconstructions of zebrafish development (Huisken et al., 2004). In this regard, compared to deSPIM, rmSPIM trades temporal resolution for spatial resolution. This modality incorporates one imaging objective, one excitation light sheet, and a rotating sample holder (see Figure 4B). rmSPIM offers up to four orthogonal and/or opposing views. By acquiring a 3D stack at each of the four locations, opposing views can

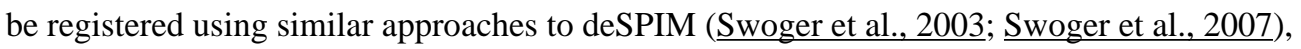
leaving two orthogonal 3D volumes to register into one final volume rendering. After deconvolution and orthogonal registration, the final rendered volume has superior resolution and contrast along with isometric voxels. While rmSPIM can provide more views, resulting in superior resolution and reduced striping artifacts, it has lower temporal resolution and precision as the sample must be rotated between acquisitions.

Finally dual-view SPIM (dvSPIM), has a lower reconstructed signal to noise, compared to rotating the sample for 4 orthogonal views, but provides greatly improved temporal resolution and precision, as the sample arrangement is unchanged between acquisitions ( $\mathrm{Wu}$ et al., 2013). Without sample manipulation, and offering most of the benefits of deSPIM, dvSPIM has the highest asset to detriment performance of the mSPIM approaches. In this setup, the sample is placed within the working distance of two identical orthogonal objectives, exactly like the basic iSPIM setup. However, in this case, each objective is capable of producing an excitation sheet and emission collection simultaneously (see Figure 4C). The two objectives work in concert to sequentially image the 3D volume from two orthogonal viewpoints. The orthogonal views are then reconstructed in the same manner as rmSPIM images. Hence, the dual-view method also generates isotropic voxels giving increased axial resolution. Since dvSPIM does not require sample manipulation to acquire orthogonal views, it is far superior to rmSPIM in terms of temporal resolution capabilities. Furthermore, dvSPIM has the potential to enhance the LSFM modality even further with unmatched dual-color imaging speeds. Since registration of two spatially different fluorescent signals is not easily achieved, dual dichroics (eg. $488 \mathrm{~nm} / 561 \mathrm{~nm}$ ), placed in the light path of each excitation/emission path, offer the capability of imaging dual color image stacks from orthogonal views resulting in dual color, isotropic, high spatio-temporal resolution 4D images. Wu et al. used this approach to successfully track microtubule tips in three-dimensions in living cells as well as improving the imaging of nuclear dynamics during C. elegans embryogenesis (Wu et al., 2013).

\section{Bessel Beam Light Sheet Fluorescence Microscopy (BB-LSFM)}

As described above, LSFM setups have the ability to capture dynamic events in living biological samples over prolonged periods of time with less photodamage or fluorophore bleaching as compared to standard confocal imaging approaches. Despite those advantages, 
those methodologies are still limited by the presence of out-of-focus excitation. LSFM using an optimized Gaussian light sheet is still 3-4 times poorer in axial resolution even when using high NA excitation and imaging objectives than point scanning confocal (LSM) and Nipkow spinning disk (SD) microscopy (Planchon et al., 2011). In addition, these systems are also limited with respect to imaging depth. To resolve the resolution and depth penetration issues, Planchon et al. employed the use of Bessel beams in order to generate thinner excitation light sheets (Gao et al., 2014; Gao et al., 2012; Planchon et al., 2011).

Bessel beams are a class of non-diffracting beams, i.e. they do not suffer from diffraction as they propagate and will maintain their relatively tight, unabberated focus over a prolonged distance $\sim 50 \mu \mathrm{m}$ (Planchon et al., 2011). The central intensity peak of the beam can be efficiently decoupled from the lower intensity shoulders which allows for self-reconstruction unlike the traditional Gaussian light sheets used in earlier LSFM setups (see Figure 5A-B) (Huisken et al., 2004; Keller et al., 2008). In order to generate a Bessel beam, a narrow annular aperture is placed in the beam path (Gao et al., 2014; Planchon et al., 2011). A true Bessel beam is impossible to generate experimentally as it has no limits and would thus require an infinite amount of energy for propagation. However, reasonably good approximations can be made that resemble a Bessel/Gaussian function shape. The distance over which this beam remains in tight focus however is finite and can be adjusted by modifying the placement of the annular aperture relative to the imaging objective (Gao et al., 2014). As in the case with the second class of LSFMs described earlier, a galvanometric mirror is used to scan the Bessel beam in the $\mathrm{x}$-axis to create the excitation sheet necessary for imaging a single optical plane. The addition of a second galvanometric mirror oscillating in the y-axis coupled with a piezoelectric element placed on the imaging objective to coordinate the image acquisition in each slice, affords the ability to move the excitation sheet, without moving the sample. This increases the image acquisition speed up to $\sim 40$ slices/s (Table 1) (Planchon et al., 2011). Despite the advantages of this approach, the intensity profile of the Bessel beam has outer-lying lobes that contain a substantial amount of excitation energy, which results in a loss of captured photons for each image plane of the sample. This results in lower axial (z) resolution and limits the efficiency and accuracy of image deconvolution.

An approach to alleviate the issues with deconvolution is to apply a periodic pattern to the excitation Bessel beam. In brief, a single image is acquired using a Bessel beam containing a periodic oscillating pattern. This pattern is then stepped in $1 / 3$ increments whilst acquiring additional images. With these three images, $I_{n}$, one can create a final imaging, $I_{\text {final }}$ according to the following relationship:

$$
I_{\text {final }}=\left|\sum_{n-1}^{N} I_{n} e^{2 \pi i n / N}\right| \text { Equation } 3
$$

where $N$ is the number of images acquired. This process removes the out-of-focus fluorescence leaving behind only the focal plane modulated information (Neil et al., 1997; Planchon et al., 2011). The reported axial resolution was at a maximum $270 \mathrm{~nm}$ at a period just below the $\lambda / \mathrm{NA}_{\text {BesselMax }}$ (see Table 1). Issues do arise with applying this algorithm to the sample as the final image has a reduced SNR (signal-to-noise ratio) due to the removal 
of considerable signal encoded in the zero frequency component of the applied modulation. In order to improve the SNR, multi-harmonic patterns can be introduced into the Bessel beam. This is achieved by stepping the beam at a fundamental period larger than $\lambda /$ $\mathrm{NA}_{\text {BesselMax}}$. To create the final image with a pattern having $H$ harmonics, Equation 3 was used again with the one exception of $N \leq H+2$. This results in slightly poorer axial resolution $(290 \mathrm{~nm})$ than the single harmonic variant described above, but provides improved SNR albeit at a slower acquisition speed with an increased number of acquisition frames per image plane (Planchon et al., 2011).

Despite these improvements, problems still exist with out-of-focus excitation. This results in lower resolution three-dimensional reconstructions, photodamage from imaging multiple harmonics of the excitation pattern at high intensities and slower imaging speeds limiting live cell imaging applications. To counter these issues, two-photon excitation has been introduced to the Bessel beam setup (Fahrbach et al., 2013; Gao et al., 2014; $\underline{\text { Planchon et al., }}$ 2011; Zhao et al., 2014). By utilizing a two-photon excitation paradigm, the outer-lying lobes of the Bessel beam no longer become relevant, as the probability for two-photon excitation diminishes by the square of the excitation intensity. As such, they no longer contribute to any out-of-focus excitation. In this case a sheet of thickness of 450nm with a continuous scanning beam can be generated. This beam is near isotropic with a continuous focal length of tens of micrometers. With the added benefit of being able to capture many tens of slices per second, it provides both a confined beam and allows fast image acquisition. The two-photon Bessel beam is very useful for imaging deeper into tissue or other specimens with greater optical aberration in comparison to both the Bessel beam and the harmonic oscillation Bessel beam systems. However, the caveats to this approach is the necessity to have a relatively high number of fluorophores in the excitation volume, a limited capacity for imaging in multiple colors by the photostability of red fluorescent proteins under two-photon excitation and the overall expense of a titanium sapphire laser as the two-photon excitation source.

Finally, to address the problems of both single/multi-harmonic and two-photon Bessel beam approaches, the use of Bessel beam Super-Resolution Structured Illumination Microscopy combines both the periodically stepped Bessel beam and the principles of 3D SuperResolution Structured Illumination Microscopy (3D SR-SIM) (Gao et al., 2014; Gao et al., 2012). The Bessel beam SR-SIM has approximately two times the axial resolution as compared to confocal techniques and roughly similar to that of widefield SR-SIM (see Table 1). It also offers reduced phototoxicity and increased SNR (Gao et al., 2012). However with thicker samples > $10 \mu \mathrm{m}$ the Bessel beam SR-SIM outperforms widefield SRSIM in both resolution and acquisition speed. Bessel beam SR-SIM is capable of up to 27 planes per second in comparison to widefield SR-SIM that acquires at a rate of 10-20 planes per second. This is simply due to the number of images involved in acquisition; the Bessel beam requires 3-5 images per plane whereas SR-SIM requires a minimum of 15 images per plane to reconstruct a super-resolution image. As such, the capabilities of this system are better suited to fast imaging in thin layers of living cells as opposed to much thicker specimens, although the ability to use conventional materials for imaging are somewhat 
limited by both the objective setup and the necessary geometrical constraints of the microscope.

\section{Stimulated Emission Depletion Selective Plane Illumination Microscopy (STED-SPIM)}

Another emerging super-resolution method using light-sheet excitation technology is the use of the stimulated emission depletion paradigm (STED-SPIM). STED is a process by which an excitation laser and then depletion laser is used to create a reduced spatial area of fluorescence emission. In the simplest case, if the depletion and excitation beams completely overlap, there will be no detectable fluorescence emission, i.e. all available fluorescence will be depleted. If however, the depletion beam is shaped in a way to allow partial excitation (i.e. in the shape of hollow donut) then fluorescence emission will occur in the spatial region where the depletion laser is not present. As intensity of the depletion laser is increased, the spatial area of the fluorescing spot is reduced allowing the breach of diffraction limit (Hell and Wichmann, 1994). The STED resolution can be described by a modified Abbe equation:

$$
\Delta r=\frac{\lambda}{2 N A \sqrt{I+I_{\text {dep }} / I_{\text {Sat }}}} \text { Equation } 4
$$

where $I_{d e p} / I_{\text {sat }} \rightarrow \infty$ implies an infinite resolution (for a single molecule). The fluorescent sample is first illuminated with the excitation light and then almost immediately illuminated with the patterned depletion beam. The short time frame between the two beams allows the fluorophores in the depletion region to emit lower-energy photons matching that of the depletion laser. This provides fluorescence in the region where the depletion laser is not patterned. Earlier STED microscopes used pulsed lasers to generate the depletion illumination pattern (Hell and Wichmann, 1994), but more recent advancements have utilized continuous wave lasers (Moneron et al., 2010; Willig et al., 2007) or supercontinuum sources (Wildanger et al., 2008) as well as time-gating approaches (Vicidomini et al., 2011). To implement this technology into an LSFM setup, a continuous wave STED beam was used with a modified optical pathway by overlapping the excitation beam with a depleting laser beam, which had a beam mode of $\mathrm{TEM}_{01}$ in the focus on either side of the excitation light sheet (see Figure 6A-B). This process serves to reduce the emission in the axial direction of the imaging plane for smaller specimen sectioning. Improvements of SPIM were seen to be $11-40 \%$ in the lateral resolution and $46 \%$ in the axial plane (Friedrich et al., 2011). The ability to resolve at higher spatial resolution has been applied to visualize the actin network in zebrafish embryos with ATTO647 conjugated phalloidin. This allows for capabilities of higher degree of resolution and at acquisition speeds of 2 to 10 frames/second (Friedrich et al., 2011).

There are however limitations to STED-SPIM as there are with traditional STED implementations that center on which fluorophores can be used successfully for depletion. They must have high quantum efficiency for fluorescence and be photostable under the high powers necessary for efficient depletion. Furthermore there are limitations to the 
multiplexing of fluorophores as they must be far apart in the visible spectrum to be effectively imaged at the same time.

\section{Other Light Sheet Based Imaging Approaches}

Other advancements of LSFM including super-resolution techniques have recently been developed and utilized in studies to increase resolution, acquisition speed, or both. Bessel beam STED-SPIM incorporates the two technologies to reduce the laser power necessary to illuminate the sample and to create a thinner sheet compared to STED-SPIM (Zhang et al., 2014). Though these studies have been limited in scope, they show promising results demonstrating the potential for faster acquisition speeds in the live cell imaging of localized signal. Two photon SR-LSFM has also been conducted without the use of Bessel beams and shows sampling depth as great as $800 \mu \mathrm{m}$ with low distortions and high localization precision (Cella Zanacchi et al., 2013). They were able to perform localization of $\mathrm{pH} 2 \mathrm{AX}$ a phosophohistone in NB4 cells with a resolution of $40 \mathrm{~nm}$. Hu et al. developed a Light-Sheet Bayesian Super-Resolution Microscope (LSBM) for the detection of nanoscopic features of heterochromatin in both live and fixed cells. LSBM provides both increases in temporal and lateral resolution (50-60 $\mathrm{nm}$ in $2.3 \mathrm{~s}$ ) ( $\mathrm{Hu}$ et al., 2013). Gebhardt et al. have developed a reflected light-sheet microscope (RLSM) where an elliptical beam parallel to the sample is reflected to the sample (Gebhardt et al., 2013). This was shown to increase Signal-to-oise ratio in samples of DNA-bound to the glucocorticoid receptor (GR) and measure residence times of the GR and an estrogen receptor-a. This also has advantages in the setup as commercial inverted microscopes can be used to create a LSFM.

\section{Concluding remarks}

Despite the concept of LSFM imaging being reported over a century ago, it has only been in the last decade that the biological applications have been truly realized. Despite the primary application for light sheet imaging being initially identified as studying long-term developmental processes such as embryogenesis, multiple research groups have developed both novel and innovative variations on the technique in terms of imaging orientation and the addition of super-resolution excitation paradigms that have enabled the imaging of dynamic behavior of biological systems at and beyond the diffraction limit. These new tools and technologies have drastically increased not only the type of samples that can be imaged, but also the spatial and temporal resolutions that can be accessed. Now, what was originally thought to be a boon for developmental biologists is turning into an innovative tool for cell biologists to study cellular and sub-cellular dynamics and neuroscientists to look at highspeed neural activity in the intact animal brains.

Notwithstanding there being multiple different types of light sheet based approaches available to choose from, researchers from multiple fields within the life sciences will clearly benefit from having more gentle and high-speed 4D imaging tools at their disposal. As this review has illustrated, each of the described techniques has advantages and limitations regarding time and length scales as well as sample orientation and preparation suggesting careful consideration when deciding on an approach that will prove to be most useful for the biological question under consideration. As with all advances in light

Curr Protoc Cytom. Author manuscript; available in PMC 2016 January 05. 
microscopy to date, future research will continue to serve as a driver for technology development, which in turn will facilitate the types of experiments that push the boundaries of what we can visualize.

\section{Acknowledgements}

We gratefully acknowledge financial support from the Waitt Advanced Biophotonics Center (AFL, SED and JAJF), NCI P30 Cancer Center Support Grant CA014195-39 (MWA, SED and JAJF), NINDS P30 Neuroscience Center Core Grant NS072031-01A1 (MSJ and JAJF) and the W.M. Keck Foundation (MWA, MSJ and JAJF). The authors also gratefully acknowledge Sreekanth Chalasani for critical reading of the manuscript and for helpful suggestions regarding organization and formatting.

\section{Literature Cited}

Ahrens MB, Orger MB, Robson DN, Li JM, Keller PJ. Whole-brain functional imaging at cellular resolution using light-sheet microscopy. Nature methods. 2013; 10:413-420. [PubMed: 23524393]

Becker W, Su B, Holub O, Weisshart K. FLIM and FCS detection in laser-scanning microscopes: increased efficiency by GaAsP hybrid detectors. Microscopy research and technique. 2011; 74:804811. [PubMed: 23939667]

Buytaert JA, Descamps E, Adriaens D, Dirckx JJ. The OPFOS Microscopy Family: High-Resolution Optical Sectioning of Biomedical Specimens. Anatomy research international. 2012; 2012:206238. [PubMed: 22567307]

Cella Zanacchi F, Lavagnino Z, Faretta M, Furia L, Diaspro A. Light-sheet confined super-resolution using two-photon photoactivation. PloS one. 2013; 8:e67667. [PubMed: 23844052]

Chardes C, Melenec P, Bertrand V, Lenne PF. Setting Up a Simple Light Sheet Microscope for In Toto Imaging of C. elegans Development. Journal of visualized experiments : JoVE. 2014

Fahrbach FO, Gurchenkov V, Alessandri K, Nassoy P, Rohrbach A. Light-sheet microscopy in thick media using scanned Bessel beams and two-photon fluorescence excitation. Optics express. 2013; 21:13824-13839. [PubMed: 23736637]

Friedrich M, Gan Q, Ermolayev V, Harms GS. STED-SPIM: Stimulated emission depletion improves sheet illumination microscopy resolution. Biophysical journal. 2011; 100:L43-45. [PubMed: 21504720]

Fuchs E, Jaffe J, Long R, Azam F. Thin laser light sheet microscope for microbial oceanography. Optics express. 2002; 10:145-154. [PubMed: 19424342]

Gao L, Shao L, Chen BC, Betzig E. 3D live fluorescence imaging of cellular dynamics using Bessel beam plane illumination microscopy. Nature protocols. 2014; 9:1083-1101.

Gao L, Shao L, Higgins CD, Poulton JS, Peifer M, Davidson MW, Wu X, Goldstein B, Betzig E. Noninvasive imaging beyond the diffraction limit of 3D dynamics in thickly fluorescent specimens. Cell. 2012; 151:1370-1385. [PubMed: 23217717]

Gebhardt JC, Suter DM, Roy R, Zhao ZW, Chapman AR, Basu S, Maniatis T, Xie XS. Singlemolecule imaging of transcription factor binding to DNA in live mammalian cells. Nature methods. 2013; 10:421-426. [PubMed: 23524394]

Hanley QS, Verveer PJ, Gemkow MJ, Arndt-Jovin D, Jovin TM. An optical sectioning programmable array microscope implemented with a digital micromirror device. Journal of microscopy. 1999; 196:317-331. [PubMed: 10594772]

Hell SW, Wichmann J. Breaking the diffraction resolution limit by stimulated emission: stimulatedemission-depletion fluorescence microscopy. Optics letters. 1994; 19:780-782. [PubMed: 19844443]

Hendriks BH, Bierhoff WC, Horikx JJ, Desjardins AE, Hezemans CA, t Hooft GW, Lucassen GW, Mihajlovic N. High-resolution resonant and nonresonant fiber-scanning confocal microscope. Journal of biomedical optics. 2011; 16:026007. [PubMed: 21361691]

Hu YS, Zhu Q, Elkins K, Tse K, Li Y, Fitzpatrick JA, Verma IM, Cang H. Light-sheet Bayesian microscopy enables deep-cell super-resolution imaging of heterochromatin in live human embryonic stem cells. Optical Nanoscopy. 2013; 2 
Huisken J, Stainier DY. Even fluorescence excitation by multidirectional selective plane illumination microscopy (mSPIM). Optics letters. 2007; 32:2608-2610. [PubMed: 17767321]

Huisken J, Swoger J, Del Bene F, Wittbrodt J, Stelzer EH. Optical sectioning deep inside live embryos by selective plane illumination microscopy. Science. 2004; 305:1007-1009. [PubMed: 15310904]

Kaufmann A, Mickoleit M, Weber M, Huisken J. Multilayer mounting enables long-term imaging of zebrafish development in a light sheet microscope. Development. 2012; 139:3242-3247. [PubMed: 22872089]

Keller PJ, Schmidt AD, Wittbrodt J, Stelzer EH. Reconstruction of zebrafish early embryonic development by scanned light sheet microscopy. Science. 2008; 322:1065-1069. [PubMed: 18845710]

Keller PJ, Stelzer EH. Quantitative in vivo imaging of entire embryos with Digital Scanned Laser Light Sheet Fluorescence Microscopy. Current opinion in neurobiology. 2008; 18:624-632. [PubMed: 19375303]

Lorenzo C, Frongia C, Jorand R, Fehrenbach J, Weiss P, Maandhui A, Gay G, Ducommun B, Lobjois $\mathrm{V}$. Live cell division dynamics monitoring in 3D large spheroid tumor models using light sheet microscopy. Cell division. 2011; 6:22. [PubMed: 22152157]

Michalet X, Cheng A, Antelman J, Suyama M, Arisaka K, Weiss S. Hybrid photodetector for singlemolecule spectroscopy and microscopy. Proceedings -Society of Photo-Optical Instrumentation Engineers. 2008:6862.

Moneron G, Medda R, Hein B, Giske A, Westphal V, Hell SW. Fast STED microscopy with continuous wave fiber lasers. Optics express. 2010; 18:1302-1309. [PubMed: 20173956]

Nakano A. Spinning-disk confocal microscopy --a cutting-edge tool for imaging of membrane traffic. Cell structure and function. 2002; 27:349-355. [PubMed: 12502889]

Neil MA, Juskaitis R, Wilson T. Method of obtaining optical sectioning by using structured light in a conventional microscope. Optics letters. 1997; 22:1905-1907. [PubMed: 18188403]

Pampaloni F, Ansari N, Stelzer EH. High-resolution deep imaging of live cellular spheroids with lightsheet-based fluorescence microscopy. Cell and tissue research. 2013; 352:161-177. [PubMed: 23443300]

Planchon TA, Gao L, Milkie DE, Davidson MW, Galbraith JA, Galbraith CG, Betzig E. Rapid threedimensional isotropic imaging of living cells using Bessel beam plane illumination. Nature methods. 2011; 8:417-423. [PubMed: 21378978]

Santi PA, Johnson SB, Hillenbrand M, GrandPre PZ, Glass TJ, Leger JR. Thin-sheet laser imaging microscopy for optical sectioning of thick tissues. BioTechniques. 2009; 46:287-294. [PubMed: 19450235]

Siedentopf H, Zsigmondy R. Uber Sichtbarmachung und Größenbestimmung ultramikoskopischer Teilchen, mit besonderer Anwendung auf Goldrubingläser. Annalen der Physik. 1902; 315:1-39.

Strobl F, Stelzer EH. Non-invasive long-term fluorescence live imaging of Tribolium castaneum embryos. Development. 2014; 141:2331-2338. [PubMed: 24803590]

Swoger J, Huisken J, Stelzer EH. Multiple imaging axis microscopy improves resolution for thicksample applications. Optics letters. 2003; 28:1654-1656. [PubMed: 13677526]

Swoger J, Muzzopappa M, Lopez-Schier H, Sharpe J. 4D retrospective lineage tracing using SPIM for zebrafish organogenesis studies. Journal of biophotonics. 2011; 4:122-134. [PubMed: 20925108]

Swoger J, Verveer P, Greger K, Huisken J, Stelzer EH. Multi-view image fusion improves resolution in three-dimensional microscopy. Optics express. 2007; 15:8029-8042. [PubMed: 19547131]

Takao D, Taniguchi A, Takeda T, Sonobe S, Nonaka S. High-speed imaging of amoeboid movements using light-sheet microscopy. PloS one. 2012; 7:e50846. [PubMed: 23227214]

Taormina MJ, Jemielita M, Stephens WZ, Burns AR, Troll JV, Parthasarathy R, Guillemin K. Investigating bacterial-animal symbioses with light sheet microscopy. The Biological bulletin. 2012; 223:7-20. [PubMed: 22983029]

Verveer PJ, Swoger J, Pampaloni F, Greger K, Marcello M, Stelzer EH. High-resolution threedimensional imaging of large specimens with light sheet-based microscopy. Nature methods. 2007; 4:311-313. [PubMed: 17339847]

Curr Protoc Cytom. Author manuscript; available in PMC 2016 January 05. 
Vicidomini G, Moneron G, Han KY, Westphal V, Ta H, Reuss M, Engelhardt J, Eggeling C, Hell SW. Sharper low-power STED nanoscopy by time gating. Nature methods. 2011; 8:571-573. [PubMed: 21642963]

Voie AH, Burns DH, Spelman FA. Orthogonal-plane fluorescence optical sectioning: threedimensional imaging of macroscopic biological specimens. Journal of microscopy. 1993; 170:229-236. [PubMed: 8371260]

Wildanger D, Rittweger E, Kastrup L, Hell SW. STED microscopy with a supercontinuum laser source. Optics express. 2008; 16:9614-9621. [PubMed: 18575529]

Willig KI, Harke B, Medda R, Hell SW. STED microscopy with continuous wave beams. Nature methods. 2007; 4:915-918. [PubMed: 17952088]

Wu Y, Ghitani A, Christensen R, Santella A, Du Z, Rondeau G, Bao Z, Colon-Ramos D, Shroff H. Inverted selective plane illumination microscopy (iSPIM) enables coupled cell identity lineaging and neurodevelopmental imaging in Caenorhabditis elegans. Proceedings of the National Academy of Sciences of the United States of America. 2011; 108:17708-17713. [PubMed: 22006307]

Wu Y, Wawrzusin P, Senseney J, Fischer RS, Christensen R, Santella A, York AG, Winter PW, Waterman CM, Bao Z, Colon-Ramos DA, McAuliffe M, Shroff H. Spatially isotropic fourdimensional imaging with dual-view plane illumination microscopy. Nature biotechnology. 2013; 31:1032-1038.

Zhang P, Goodwin PM, Werner JH. Fast, super resolution imaging via Bessel-beam stimulated emission depletion microscopy. Optics express. 2014; 22:12398-12409. [PubMed: 24921358]

Zhao M, Zhang H, Li Y, Ashok A, Liang R, Zhou W, Peng L. Cellular imaging of deep organ using two-photon Bessel light-sheet nonlinear structured illumination microscopy. Biomedical optics express. 2014; 5:1296-1308. [PubMed: 24876996] 


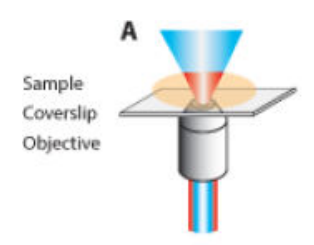

Wide Field

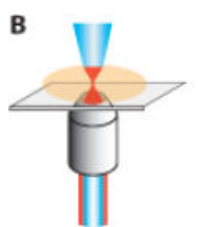

Laser Scanning

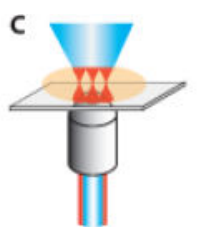

Spinning Disk
D

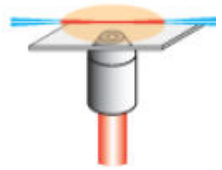

LSFM

Figure 1.

Comparison of different fluorescence imaging modalities. A. Wide field fluorescence where excitation light is focused into a sample and fluorescence is epi-collected. B. Laser scanning confocal where an excitation laser is scanned in $\mathrm{x}$ and $\mathrm{y}$ dimensions in a sample and fluorescence is epi-collected. C. Spinning disk confocal where two co-rotating disks (one of microlenses and one of pinhole apertures) scan an array of laser beamlets across the sample at high revolutions and fluorescence is epi-collected and D. Light Sheet Fluorescence Microscopy (LSFM) where an excitation laser is focussed into the sample from an orthogonal direction and fluorescence is collected by a separate imaging objective. 


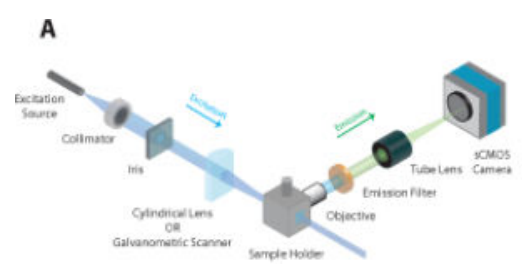

B

C

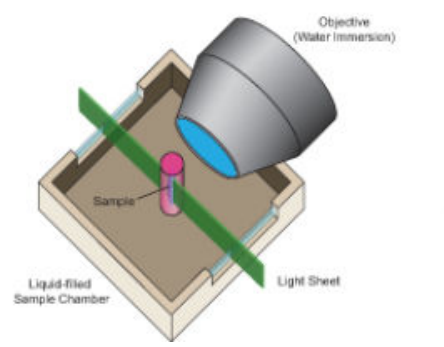

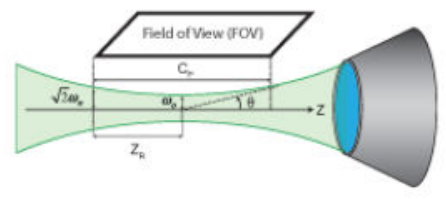

D

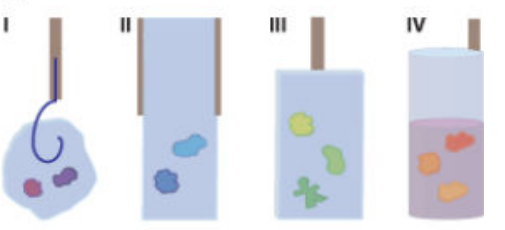

Figure 2.

Basic Light Sheet Fluorescence Microscope (LSFM). A. Schematic design of the basic light sheet fluorescence microscope. An excitation laser is collimated, passed through an iris and either focused into a sheet via a low NA cylindrical lens or scanned into a sheet via a galvanometric scanning mirror. The excitation light sheet passes through the sample in an appropriate imaging chamber and fluorescence is collected orthogonally by an imaging microscope objective and imaged through a tube lens onto a sCMOS camera. B. Schematic dimensions of the light sheet beam waist and how they relate to the field of view (FOV). C. Cut away schematic diagram of the sample-imaging chamber. The sample (symmetric about two-axes) is mounted in the center in a support medium such as agarose on either a translating, rotating or combination mount. The imaging objective (water-immersion) fits into this chamber which is filled with an immersion suitable liquid. D. Samples in basic LSFM are usually suspended by gravity in agarose in an immersion filled sample chamber. The strategies for supporting the agarose mounted samples range from I. a simple hook into the gel, II. a more sophisticated sculpted sample holder, III. a support basket and IV. a cylinder filled with growth media for cultured cells. 


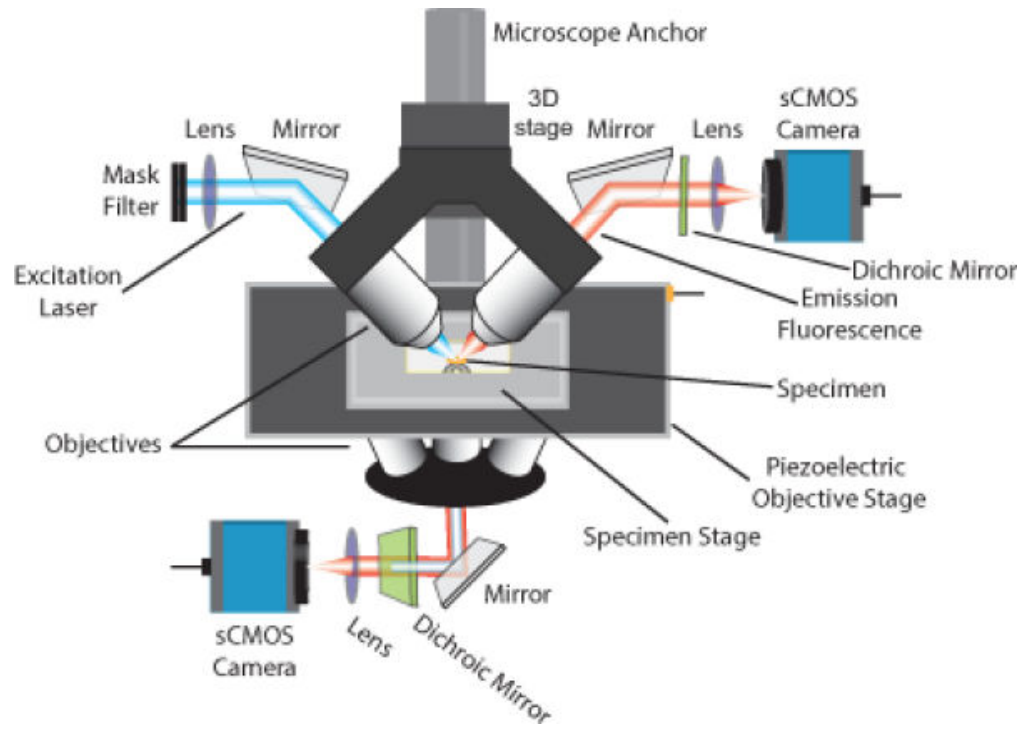

Figure 3.

Inverted Selective Plane Illumination Microscope. An excitation laser passes through a mask and is reflected using a mirror into a $45^{\circ}$ oriented excitation objective mounted on an anchor pillar which directs the excitation light sheet into the sample. A second $45^{\circ}$ oriented imaging objective collects the fluorescence, which then passes through an appropriate emission filter and is imaged through a tube lens on an sCMOS camera. A second sCMOS camera is available under the standard inverted microscope platform objectives for focus finding and transmitted light purposes. 
A

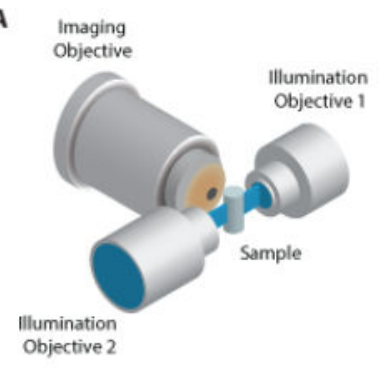

B

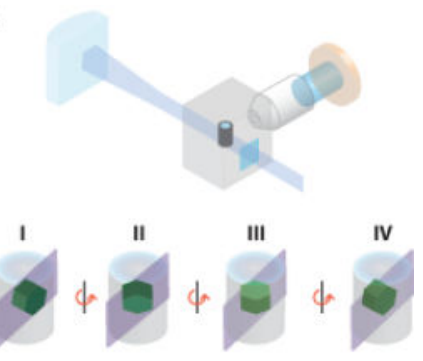

c

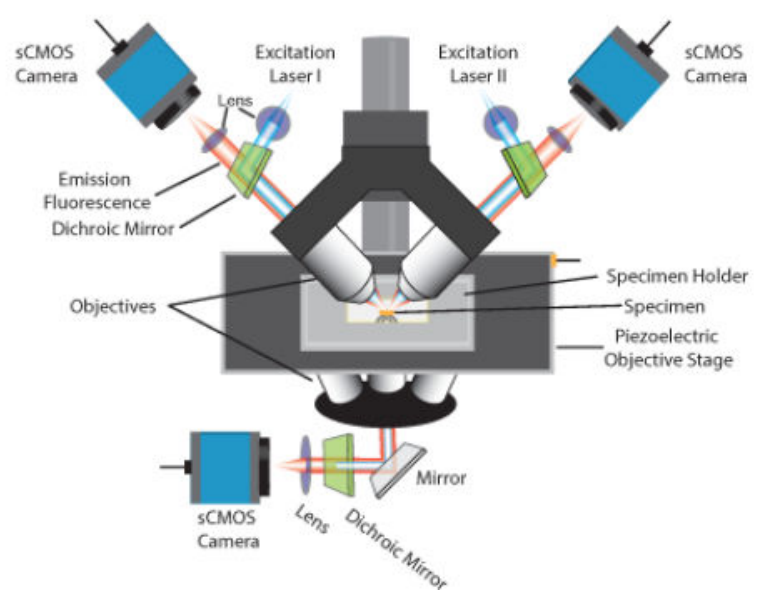

Figure 4.

Multi-view Selective Plane Illumination Microscopes. A. Dual-view Selective Plane Illumination Microscope (dvSPIM). Two illumination objective lenses are placed at $180^{\circ}$ orientations to one another with respect to a third single imaging objective orthogonal to the excitation plane. B. Rotation Multi-view Selection Plane Illumination Microscope (rmSPIM). Much like the basic LSFM setup a cylindrical lens focuses an excitation light sheet into a sample mounted in a suitable imaging chamber. In this case, the sample is mounted onto a combination rotation and translation stage. I. a z-stack is taken at the first orientation, II. a second z-stack is taken at an orientation $90^{\circ}$ perpendicular to the first, III. A third z-stack is taken $90^{\circ}$ perpendicular to the second and IV. a final z-stack is taken $90^{\circ}$ perpendicular to the third. All four z-stacks are then recombined and deconvolved to reconstruct a three-dimensional high-resolution image. C. Dual-view Selective Plane Illumination Microscope (dvSPIM). Similar to the iSPIM scenario detailed in Figure 3, this variation has excitation light sheets passing through both objectives and fluorescence emission being collected through both objectives and being imaged through an appropriate emission filter and tube lens onto a sCMOS camera. 
A

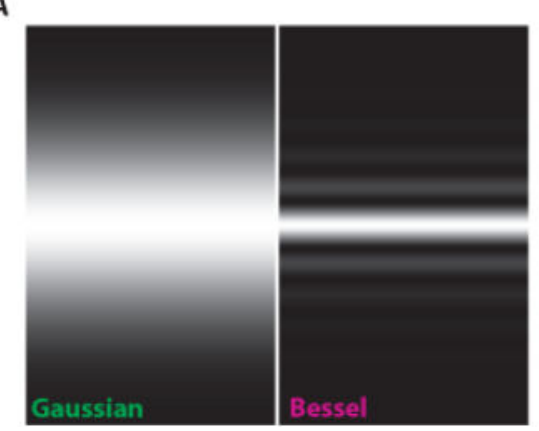

B

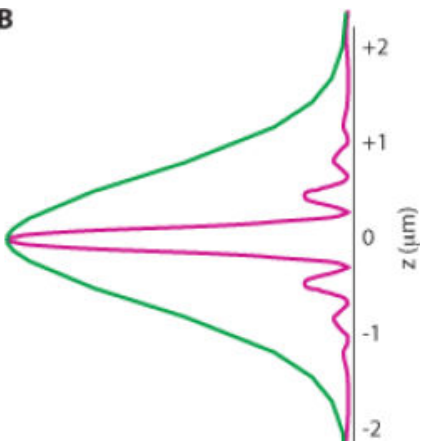

Figure 5.

Bessel beam Light Sheets. A. Left panel illustrates the xz profile of a Gaussian light sheet and right panel illustrates the xz profile of a Bessel beam light sheet. B. Line profile through the center of the xz profiles of both the Gaussian (blue) and Bessel (purple) light sheets. 

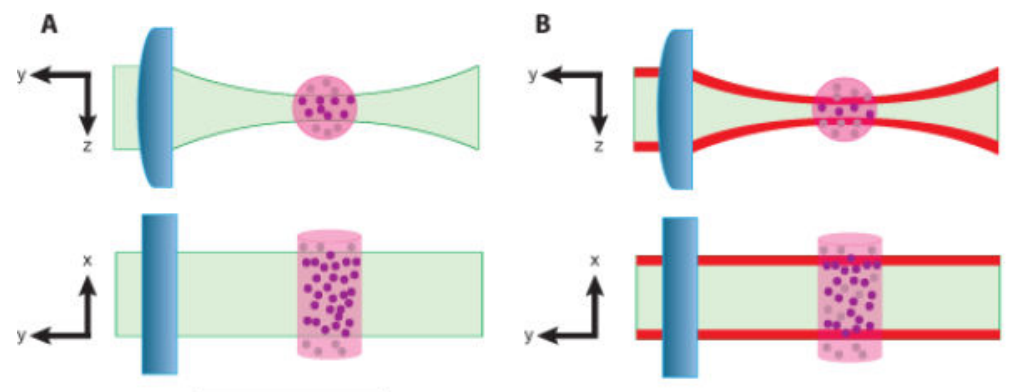

C
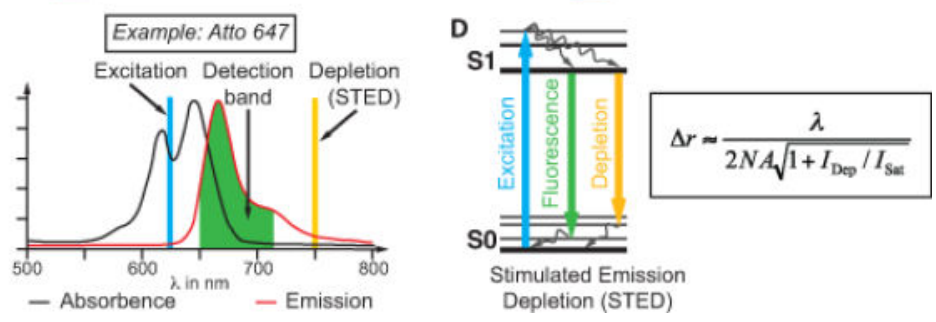

Figure 6.

STED-Selective Plane Illumination Microscope. A. Excitation optics and sample fluorescence of SPIM. B. Excitation optics and sample fluorescence of STED-SPIM (bottom). The purple spheres represent fluorescent objects whereas the grey spheres represent dark (non-fluorescing objects). The green region of the light sheet demonstrates SPIM excitation and the red regions demonstrate the regions in which STED is occurring. $\mathbf{C}$. Example spectra of the fluorophore Atto647N. Excitation is optimal at $635 \mathrm{~nm}$ and depletion at $750 \mathrm{~nm}$. Fluorescence detection is spectrally gated to occur in the spectral region 650 to $720 \mathrm{~nm}$ and thus ignores the depleted fluorescence. D. Schematic of the fluorophore electronic transitions occurring during the STED process. 


\section{Table 1}

Comparison of different Bessel beam based light sheet characteristics.

\begin{tabular}{lllll}
\hline & Bessel beam & Bessel beam Harmonics & TP Bessel beam & Bessel beam SR-SIM \\
\hline Average Spatial Resolution & $\mathrm{x} \sim 230 \mathrm{~nm}$ & $\mathrm{x} \sim 230 \mathrm{~nm}$ & $\mathrm{x} \sim 230 \mathrm{~nm}$ & $\mathrm{x} \sim 180 \mathrm{~nm}$ \\
& $\mathrm{y} \sim 230 \mathrm{~nm}$ & $\mathrm{y} \sim 230 \mathrm{~nm}$ & $\mathrm{y} \sim 230 \mathrm{~nm}$ & $\mathrm{y} \sim 230 \mathrm{~nm}$ \\
& $\mathrm{z} \sim 600 \mathrm{~nm}$ & $\mathrm{z} \sim 270-290 \mathrm{~nm}$ & $\mathrm{z} \sim 450 \mathrm{~nm}$ & $\mathrm{z} \sim 350 \mathrm{~nm}$ \\
\hline Optical Sectioning Capabilities & Fair & Fair & Excellent & \multirow{2}{*}{ Good } \\
\hline Speed & $\sim 40$ planes/s & $10-20$ planes/s & $\sim>40$ planes/s & $\sim 10-30$ planes $/ \mathrm{s}$ \\
\hline Multicolor imaging & Yes & Yes & No & \multirow{2}{*}{ Yes } \\
\hline Fluorophore Brightness Requirement & Low & Low & High & \multirow{2}{*}{ Low } \\
\hline Photodamage & Low & Fair & High & \multirow{2}{*}{ Fair } \\
\hline
\end{tabular}

